\title{
Elucidation of the anti-hyperammonemic mechanism of Lactobacillus amylovorus JBD401 by comparative genomic analysis
}

Parul Singh ${ }^{1 \dagger}$, Hea-Jong Chung ${ }^{1+}$, In-Ah Lee ${ }^{3}$, Roshan D'Souza', Hyeon-Jin Kim² and Seong-Tshool Hong ${ }^{1 *}$

\begin{abstract}
Background: Recent experimental evidence showed that lactobacilli could be used as potential therapeutic agents for hyperammonemia. However, lack of understanding on how lactobacilli reduce blood ammonia levels limits application of lactobacilli to treat hyperammonemia.

Results: We report the finished and annotated genome sequence of L. amylovorus JBD401 (GenBank accession no. CP012389). L. amylovorus JBD401 reducing blood ammonia levels dramatically was identified by high-throughput screening of several thousand probiotic strains both within and across Lactobacillus species in vitro. Administration of L. amylovorus JBD401 to hyperammonemia-induced mice reduced the blood ammonia levels of the mice to the normal range. Genome sequencing showed that L. amylovorus JBD401 had a circular chromosome of 1,946,267 bp with an average GC content of 38.13\%. Comparative analysis of the L. amylovorus JBD401 genome with $L$. acidophilus and L. amylovorus strains showed that L. amylovorus JBD401 possessed genes for ammonia assimilation into various amino acids and polyamines Interestingly, the genome of L. amylovorus JBD401 contained unusually large number of various pseudogenes suggesting an active stage of evolution.
\end{abstract}

Conclusions: L. amylovorus JBD401 has genes for assimilation of free ammonia into various amino acids and polyamines which results in removal of free ammonia in intestinal lumen to reduce the blood ammonia levels in the host. This work explains the mechanism of how probiotics reduce blood ammonia levels.

Keywords: Hyperammonemia, Lactobacillus amylovorus JBD401, Comparative genome analysis, Ammonia assimilation

\section{Background}

Ammonia is produced as a byproduct from the metabolism of nitrogenous compounds such as amino acids, purine/pyrimidine bases, and amines in mammals. Because ammonia is a strong neurotoxin, it is converted to urea in the liver prior to excretion in urine [1]. Hyperammonemia is induced when the blood concentration of ammonia is elevated as the result of a disturbance in ammonia metabolism. The induction of hyperammonemia can be congenital, resulting from a genetic defect in the enzymes in the urea cycle, or acquired, resulting from liver failure involving the urea cycle [2]. The

\footnotetext{
* Correspondence: seonghong@chonbuk.ac.kr

${ }^{\dagger}$ Equal contributors

'Department of Biomedical Sciences and Institute for Medical Science, Chonbuk National University Medical School, Jeonju, Chonbuk 54907, South Korea

Full list of author information is available at the end of the article
}

neurotoxic nature of ammonia leads hyperammonemia to result in encephalopathy, which is characterized by vomiting, hypotonia, lethargy, seizures, and coma [3]. These serious clinical consequences of hyperammonemia have motivated various approaches to reduce the blood concentration of ammonia by limited protein intake, administration of lactulose or neomycin, hemodialysis, plasmapheresis, and gut cleansing [4]. However, these approaches are not very effective in the treatment of hyperammonemia and can be used only in an emergency situation because of the complexity of the treatments.

Previous research on association between ammonia levels and Alzheimer's disease (AD) had found that the blood ammonia levels in $\mathrm{AD}$ patients were significantly higher than in control groups $[5,6]$, indicating that the neurotoxic nature of ammonia contribute to the etiology of $\mathrm{AD}$. The possible etiological association of ammonia to $\mathrm{AD}$ was further solidated by finding that Mediterrian diet which consists of 
an unusally large quantity of lactobacilli prevented AD significantly $[7,8]$. Considering the neurotoxic nature of ammonia, a positive correlation between blood ammonia levels and $\mathrm{AD}$ is not a surprising phenomenon.

The intestine is the site where digested nutrients are absorbed through intestinal capillaries and lymphatic vessels [9]. However, the metabolites do not always flow in one direction, i.e., from the intestinal lumen to intestinal capillaries or lymphatic vessels [10]. Ammonia is an example of a metabolite that does not move in a single direction; the intestine secretes and absorbs ammonia through both passive diffusion and specific transporters such as RhBG (Rh family, B glycoprotein) and RhCG (Rh family, C glycoprotein) [11]. Although not widely recognized, the intestine is a major site of ammonia transport [12]. Because ammonia can move bidirectionally between the intestinal lumen and body fluid, removal of ammonia in the intestinal lumen by intestinal microbes such as Lactobacillus species could reduce the blood concentration of ammonia, thus providing an ideal treatment method for hyperammonemia.

Although it is a promising approach, administration of lactobacilli to treat hyperammonemia [13] has not been adopted in medical practice yet. There are two problems in adopting a Lactobacillus strain as a therapeutic agent to treat hyperammonemia. The action mechanism on how lactobacilli reduce blood ammonia levels has to be established and an ideal strain for hyperammonemia needs to be identified. In this work, we screened several thousand probiotic strains both within and across Lactobacillus species and identified L. amylovorus JBD401 as a strain that most efficiently removed ammonia from the surrounding medium. Administration of $L$. amylovorus JBD401 into the hyperammonemia-induced mice reduced the blood ammonia levels to the normal range. Genome sequencing showed that L. amylovorus JBD401 has a complete set of genes required for ammonia assimilation into various amino acids and polyamines. Possession of the pathways for ammonia assimilation should enable $L$. amylovorus JBD401 to remove ammonia from the intestinal lumen, thereby eventually reducing the blood concentration of ammonia. This work not only identifies the best candidate strain for treatment of hyperammonemia, but also sheds light on the unsolved mechanism by which Lactobacillus reduce blood ammonia levels.

\section{Results}

Identification of a Lactobacillus strain, L. amylovorus JBD401, for treatment of hyperammonemia

Recent findings revealed a possibility that oral administration of certain Lactobacillus strains to hyperammonemic patients could reduce the ammonia concentration in blood [14, 15]. On the basis of these findings we extensively screened probiotic strains from the gutmicrobiotabank including Lactobacillus, Lactococcus and Streptococcus (https://www.gutmicrobiotabank.com). By high-throughput screening of several thousand probiotic strains in vitro, we identified several probiotic strains that efficiently removed ammonia from their surrounding culture medium, suggesting a possibility of development of the probiotic strains as therapeutic agents for treatment of hyperammonemia (Fig. 1a). Especially, $L$. amylovorus JBD401 was notable in terms of removing ammonia from its surrounding culture medium. In vivo experiments showed that L. amylovorus JBD401 significantly reduced the ammonia concentrations in the blood of hyperammonemic mice (Fig. 1b). The blood ammonia concentrations were decreased to half of the original values in hyperammonemia-induced mice fed L. amylovorus JBD401 for 7 days, whereas untreated hyperammonemic mice maintained abnormally high blood ammonia concentrations. In addition to reduction of blood ammonia levels, administration of $L$. amylovorus JBD401 decreased the ammonia concentrations in the brains of the hyperammonemic mice to the same extent as blood ammonia levels (Fig. 1c). These animal experiments clearly showed that $L$. amylovorus JBD401 could be developed as an ideal therapeutic agent for treatment of hyperammonemia.

It is known that ammonia causes neuronal apoptosis by activating the caspase 9-dependent apoptotic pathway [16]. To confirm whether removal of ammonia in the brain by L. amylovorus JBD401 actually inhibits ammoniainduced neurotoxicity, mouse brain tissues were analyzed using the Fluorescent-Labeled Inhibitor of Caspases $\left(\right.$ FLICA $\left.^{\mathrm{m}}\right)$ assay to detect the induction levels of caspases. As shown in Fig. 1d and Fig. 1e, the caspase 9-dependent apoptotic pathway was activated in the control hyperammonemic mice, but was not activated in the hyperammonemic mice fed L. amylovorus JBD401. Therefore, it is reasonable to conclude that $L$. amylovorus JBD401 assimilated ammonia in the mouse intestine to reduce blood ammonia levels in the hyperammonemic mice, and that feeding with $L$. amylovorus JBD401 protects neurons against the ammonia-induced neurotoxicity.

\section{Genome features of $L$. amylovorus JBD401}

After identifying $L$. amylovorus JBD401 as a potential strain for treatment of hyperammonemia, we sequenced the whole genome of $L$. amylovorus JBD401 to elucidate how this Lactobacillus strain reduces blood ammonia levels. The genome sequence of $L$. amylovorus JBD401 revealed a circular chromosome of 1,946,267 bp with an average GC content of $38.13 \%$. The complete genome sequence was deposited in the GenBank database (Accession no. CP012389). The genome contained 2244 ORFs predicted by GLIMMER, but manual annotation resulted in 1979 protein encoding genes, representing a 


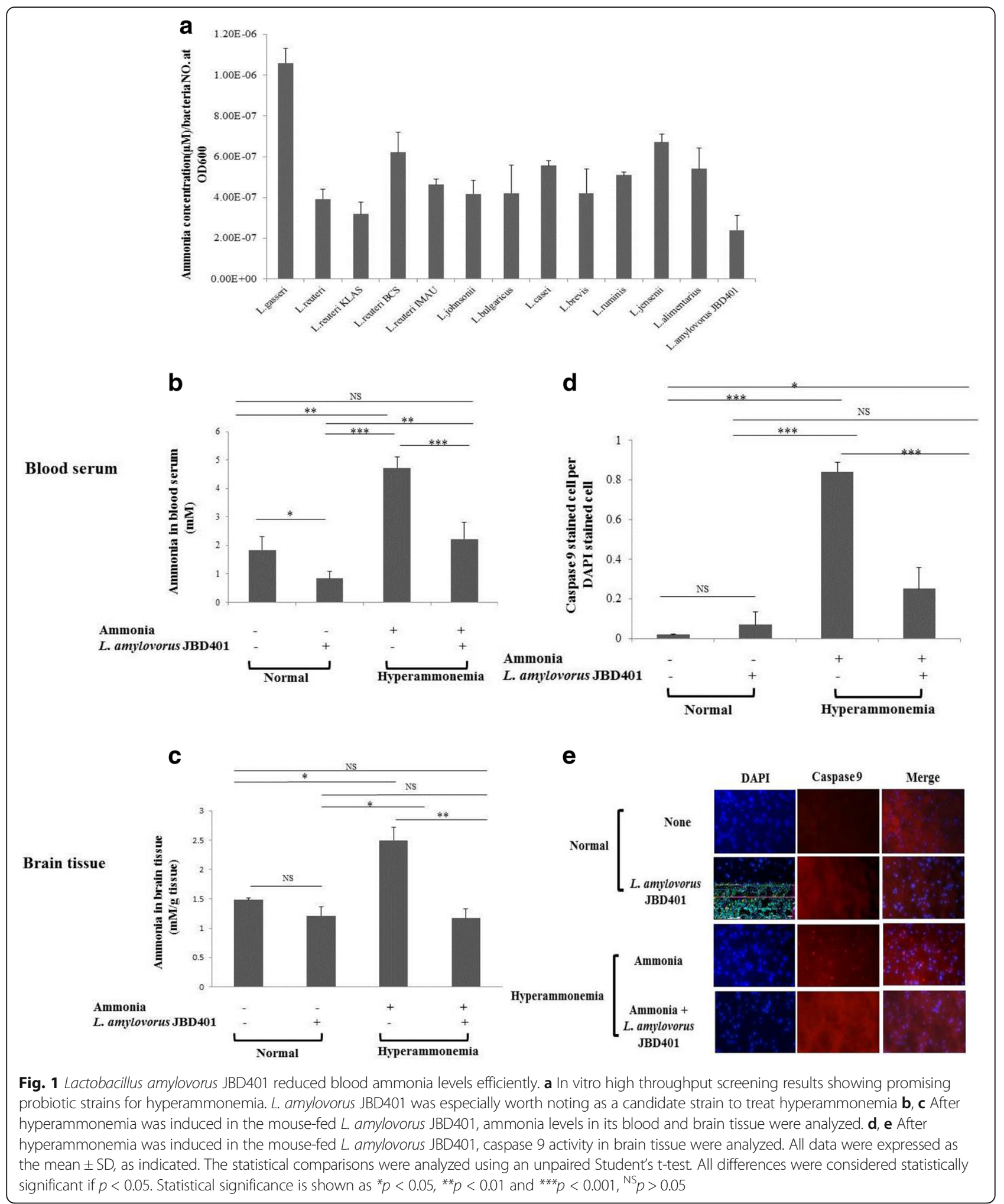

coding density of $84.17 \%$. Seven rRNAs (consisting of $5 \mathrm{~s}, 16 \mathrm{~S}$, and 23S), one non-coding RNA, and 35 tRNAs were distributed throughout the genome despite its modest size (Fig. 2).
Whole-genome sequencing of $L$. amylovorus JBD401 confirmed the presence of the complete pathway of purine metabolism with all required genes (GAR, FGAR, FGAM, AIR, CAIR, SAICAR, AICAR, FAICR, and IMP), whereas 


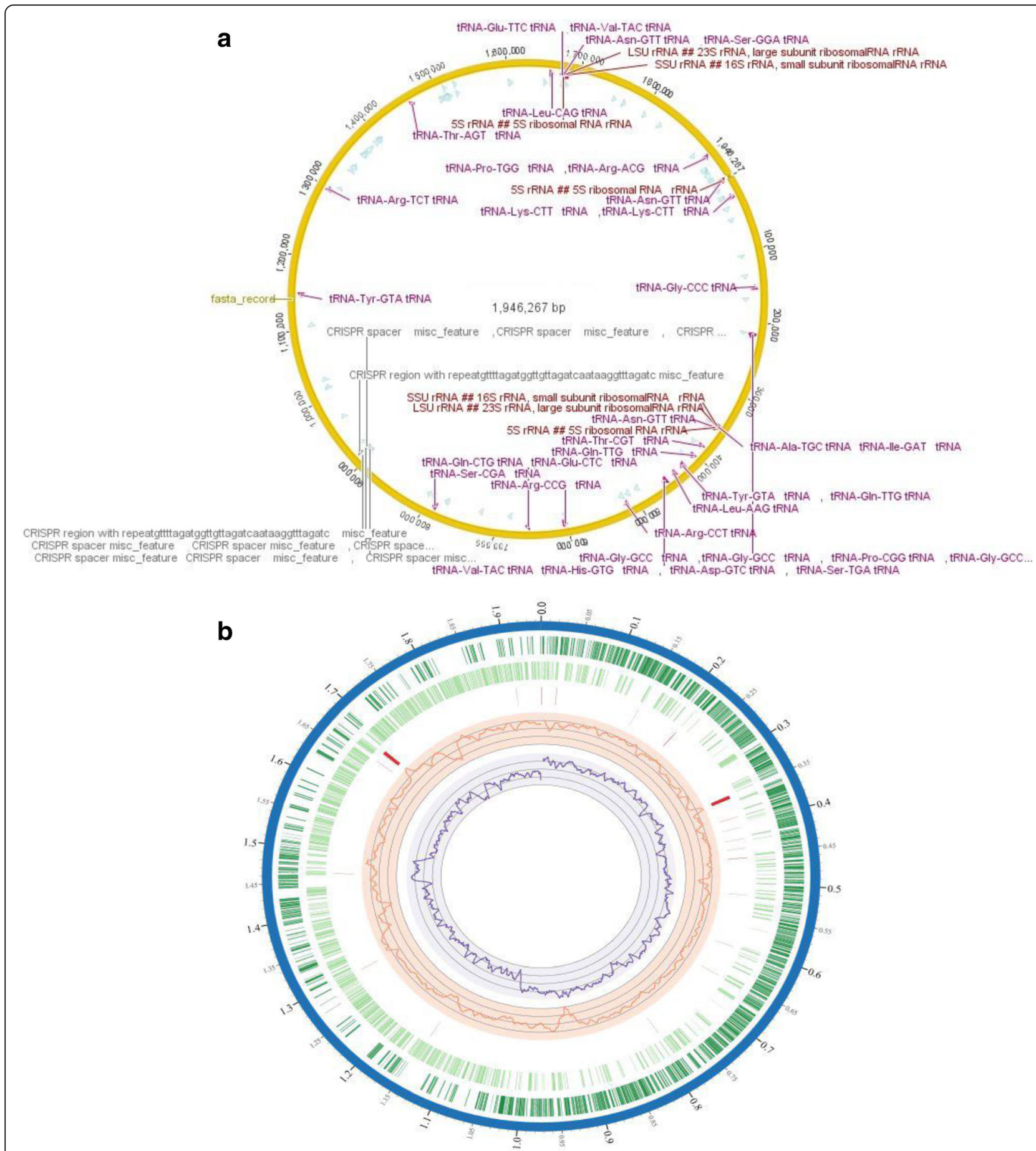

Fig. 2 Circular view of the complete genome sequence of Lactobacillus amylovorus JBD401. a The circle was created by using Geneious 7.0.6. Genome of L. amylovorus JBD401 revealed total 35 tRNAs and 7 rRNAs. Some tRNAs were redundant. Regardless of that, they represented 16 amino acid biosynthetic pathways except methionine, phenylalanine, tryptophan and cysteine. $\mathbf{b}$ Genome atlas of L. amylovorus JBD401.This atlas represents a circular view of the complete genome and was created using PATRIC. List of rings, from outside to inside 1) Chromosomes, plasmids, contigs, 2) CDS forward strand, 3) CDS reverse strand, 4) RNAs 5) GC Content line Plot and 6) GC Skew line Plot

only a partial pathway was available for pyrimidine metabolism. Two genes among 10 genes required for de novo pyrimidine metabolism were lacking. Interestingly, $L$. amylovorus JBD401 appears to assimilate ammonia through multiple pathways other than the well-known pathway of synthesizing Gln from Glu via glutamine 
synthetase I (glnA, AB283_1841). Gln synthesis seems to also be catalyzed by amidophosphoribosyltransferase (AB283_1899) upon entering the purine metabolism pathway (Additional file 1: see supplementary results "Genome features of Lactobacillus amylovorus JBD401"). L. amylovorus JBD401 possesses L-asparaginase (AB283_2020) that can synthesize Asp from Asn. The presence of asparagine synthetase (AB283_0122) in L. amylovorus JBD401 suggests that interconversion between Gln (Glutamine) and Glu (Glutamic Acid) is possible. In addition, L. amylovorus JBD401 seems to be capable of de novo synthesis of six amino acids: Asp (Aspartic Acid), Gln, Gly (Glycine), Ser (Serine), Thr (Threonine), and Cys (Cysteine). L. amylovorus JBD401 seems to be able to synthesize most, but not all, cofactors and vitamins. Overall, $L$. amylovorus JBD401 has certain degree of auxotrophy for nucleotides, amino acids, cofactors, and vitamins.

Three prophages were found in the L. amylovorus JBD401 genome, of which two were incomplete and one was intact (Fig. 3). The intact prophage showed similarity to dairy Lactobacillus helveticus bacteriophage phiAQ113 [17]. The genome of phiAQ113 phage is related to the genomes of phage KC5a (present in $L$. gasseri) and phage Lj771 (present in L. johnsonii) [18]. These phylogenetic similarities suggest a probable common ancestral origin. As expected, Virulence finder [19] did not show any virulence factors in $L$. amylovorus JBD401, indicating that L. amylovorus JBD401 is safe from outbreak of verocytotoxin-producing Escherichia coli (VTEC) infections. CRISPR genes provide immunity to the host against phage, plasmid proliferation and genetic transformation. These genes are widely found in bacteria; to date, almost $40 \%$ of bacterial genomes have been reported to contain CRISPR genes. Two CRISPR genes (Csn1 family) were identified at location 907,737908,783 bp, and two other CRISPR genes (cas1 and cas2) were present at 912098-913006 bp and 913,014913,289 bp, respectively. We recognized six bacteriocins in L. amylovorus JBD401, among which three were clustered together at AB283_27-AB283_29, and three were scattered throughout the genome at AB283_0383, AB283_1910, and AB283_2114. Among the six genes for bacteriocins, five are involved in production and processing of helveticin $\mathrm{J}$, while one encodes $\mathrm{ABC}$ transporter auxiliary bacteriocin (Additional file 1: see supplementary results "Genome features of Lactobacillus amylovorus JBD401"). We found that genome of L. amylovorus JBD401 has total 16 protein sequences that are antibiotic resistant. Among them one sequence has similarity above cutoff values (97.65\%) which is resistant to tetracycline and other 15 sequences have similarity below cutoff (Additional file 1: Table S7). We also found that carbamate kinase (CK) (EC 2.7.2.2) and ornithine carbamoyltransferase (OTC) (EC 2.1.3.3) were resistant to cephalosporin_i, penicillin and methicillin (Additional file 1: Table S8).

Generally, LAB (Lactic acid bacteria) has a limited ability to synthesize amino acids, and our genome analysis of $L$. amylovorus JBD401 showed that L. amylovorus JBD401 is no exception. Although LAB is believed to have relatively weak proteolytic systems compared to other bacteria, they are still sufficiently complex and capable of hydrolyzing proteins from food. In silico analysis of L. amylovorus JBD401 revealed the presence of a total of 53 proteases/peptidases and their related transporter proteins. $\mathrm{ABC}$ type transporters (OppA, OppB, OppC, OppD, and OppF) were identified as transporters for amino acids or oligopeptides (Additional file 1: Table S1). The proteases/peptidases of L. amylovorus JBD401 were grouped according to function and proposed family (Additional file 1: Table S2). The most dominant type of protease/peptidase was $M$ (metallo) with 14 members, followed by $\mathrm{S}$ (serine) with $12 \mathrm{mem}$ bers and $\mathrm{C}$ (cysteine) with 11 members. The group of type A (aspartic) had only 2 members, and type $\mathrm{T}$ (threonine) had just 1 member. LAB is known to be capable of using almost all carbohydrates such as mono/di/polysaccharides. Raffinose and fructooligosaccharides (FOS) are complex dietary carbohydrates that are not digested in the upper GI tract of humans [20]. The $L$. amylovorus JBD401 genome possessed nine phosphoenolpyruvate sugar-transferase systems (PTS) and some $A B C$ transporters, with six different families for FOS, ribose, maltose, sugar, and raffinose as well as some uncharacterized carbohydrates (Additional file 1: Table S3; Table S4). Interestingly, L. amylovorus JBD401 possessed genes for only partial biosynthesis of nicotinate, nicotinamide, pantothenate, CoA, folate, and retinol.

\section{Comparative genomics and functional analysis of $L$. amylovorus JBD401}

The JBD401 strain was originally classified as a strain of L. acidophilus based on its morphological and biochemical characteristics but functional annotation of JBD401 clearly indicates that the genome sequence may have originated from a strain of L. amylovorus. Subsequently, we performed average nucleotide identity (ANI) test [21] and the result suggested $L$. amylovorus rather than $L$. acidophilus for this genome. The sequence of JBD401 genome was $98.727 \%$ identical by ANI to the type genome of L. amylovorus, with $85.0 \%$ coverage of the genome. Therefore, JBD401 was reclassified as $L$. amylovorus JBD401. We compared the genome of $L$. amylovorus JBD401 with the genomes of known $L$. acidophilus strains and $L$. amylovorus strains to elucidate how L. amylovorus JBD401 reduces blood ammonia levels. Every L. acidophilus and L. amylovorus genome sequenced to date (i.e., $L$. acidophilus NCFM, $L$. 


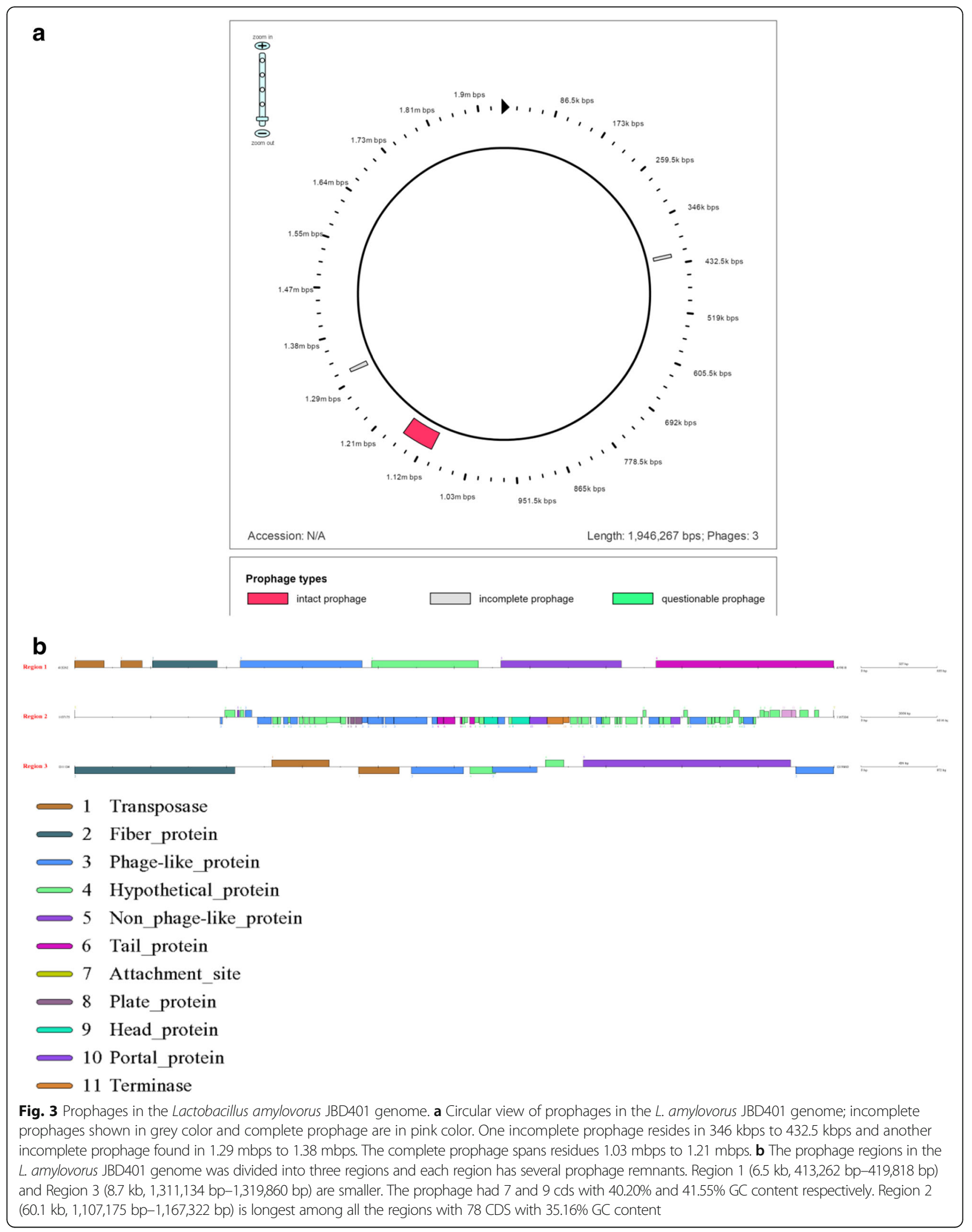


acidophilus 30SC, L. acidophilus La-14, L. acidophilus FSI4, L. amylovorus 30SC, L. amylovorus GRL1118 and L. amylovorus DSM20531) [22-26] showed similar genome size and numbers of coding genes, rRNAs, and tRNAs at the level of summary statistics (Additional file 1: Table S5; Additional file 1: Table S6). Interestingly, $L$. amylovorus JBD401 had uniquely large number of pseudogenes unlike other L. acidophilus and L. amylovorus strains. L. amylovorus JBD401 had 372 pseudogenes constituting $15.82 \%$ of the genome possibly indicative of ongoing specialization [27]. There are three main ways for pseudogenes to form in bacteria: degradation preceded by duplication, disruption by transposons and failed/incomplete horizontal gene transfer (HGT). HGT is the most common cause of pseudogenization [28].

Multiple genome alignment of $L$. amylovorus JBD401 with $L$. acidophilus NCFM, $L$. acidophilus 30SC, $L$. acidophilus FSI4, and L. acidophilus La-14 showed 55 super intervals in root alignment at the minimum weight of 15 (Fig. 4a). Overall GC content was $36.1 \%$, and root alignment length was 2,713,521 bp. A total of 542 conserved regions were found among the five genomes by manual curation and were used for further analysis. Detailed deep analysis (COREGENES) [29] of the conserved region was executed to determine core proteins. Genes with a BLASTP threshold score of 75 were considered homologs in the analysis. Interestingly, 24 of the 1200 core genes corresponded to hypothetical proteins. A total of 662 proteins in L. acidophilus NCFM, 676 proteins in L. acidophilus La-14, 837 proteins in L. acidophilus 30SC, 558 proteins in L. acidophilus FSI4, and 359 proteins in $L$. amylovorus JBD401 were uniquely present in each genome (Fig. 4b f Additional file 1: see supplementary results "Comparative genomics and functional analysis of Lactobacillus amylovorus JBD401").

We further compared genes of L. amylovorus JBD401 with previously reported genomes of $L$. amylovorus strains in a pair-wise manner of group 30SC and JBD401, group GRL1118 and JBD401 and group DSM20531 and JBD401 (Fig. 5a, b, c). The numbers of core genes in [Group 30SC and JBD401] and in [GRL1118 and JBD401] were 1391 and 1379, respectively while that was only 1318 in [DSM20531 and JBD401]. The reduced number of core genes in [DSM20531 and JBD401] suggests that L. amylovorus DSM20531 is distant to L. amylovorus JBD401. We also performed additional BLAST searches to find the evidence where $L$. amylovorus JBD401 acquired genes of our interest from (Fig. 5d, e). Interestingly, BLAST results showed that CK (EC 2.7.2.2) was found in L. amylovorus JBD401 (100\%), L. amylovorus GRL1118 (99. 89\%), L. amylovorus 30SC (99.89\%), L. amylovorus DSM20531 (99.57\%) and Pyrodictium delaneyi Su06 (100\%) (Fig. 5d). OTC (EC 2.1.3.3) was found in $L$. amylovorus JBD401 (100\%), L. amylovorus GRL1118 (99.71\%), L. amylovorus 30SC (99.71\%), L. amylovorus DSM20531 (98.84\%), Vagococcus penaei CD276 (72. 41\%) and Acetobacterium woodii DSM1030 (83.33\%) (Fig. 5e). BLAST alignment results suggest that CK and OTC genes are specific to amylovorus species in Lactobacillus. Rest of the important genes such as Nacyl-L-amino acid amidohydrolase (EC 3.5.1.14), Nacetyl-L,L-diaminopimelate aminotransferase (EC 2.6.1) , glutamine synthetase type I (EC 6.3.1.2), aspartate aminotransferase (EC 2.6.1.1) and ornithine decarboxylase (EC 4.1.1.17) were also subjected to BLAST analysis to understand the regulation of biosynthetic pathways for various amino acids and polyamine metabolites in L. amylovorus starins (Additional file 1: Figure S3; Figure S4; Figure S5).

Functional annotation of L. amylovorus JBD401 was performed by the Blast2go (basic) tool [30]. Assigned controlled vocabularies or functional terms to the gene products were represented as a combined graph including summarized filtered pie charts (Additional file 1: Figure S6). Since the combined graph was too large for the presentation of functional data, it was divided into three categories: molecular function (MF), cellular component (CC), and biological process (BP). Annotation configuration was chosen with an e-value of $1 \mathrm{e}-4$, which means that all sequences obtained with e-values higher than 1e-4 during blast were disregarded (Additional file 1: Figure S7).

\section{Identification of the complete set of genes for ammonia} assimilation in L. amylovorus JBD401 to reduce blood levels Comparative genome analysis showed that the genome of L. amylovorus JBD401 possessed the enzymes required for assimilation of ammonia: $\mathrm{CK}$ (EC 2.7.2.2) at AB283_06100 and OTC (EC 2.1.3.3) at AB283_ 06105. CK can synthesize carbamoyl phosphate from bicarbonate, ammonia, and ATP. The carbamoyl phosphate biosynthesized from ammonia can be converted into L-ornithine by OTC. L-ornithine is the key precursor metabolite for biosynthesis of various amino acids and polyamine compounds (Fig. 6). In addition to possession of the enzymes for biosynthesis of L-ornithine from ammonia, L. amylovorus JBD401 also possessed all of the enzymes for subsequent synthesis of various amino acids and polyamine compounds. This indicates that L. amylovorus JBD401 can actively absorb ammonia from its surrounding environment, i.e., intestinal fluids, to assimilate ammonia into various amino acids and polyamine compounds. Active absorption of ammonia in intestinal fluids will reduce ammonia levels in intestinal fluids so that blood ammonia will traverse to intestinal fluids to reduce blood ammonia levels. 
a

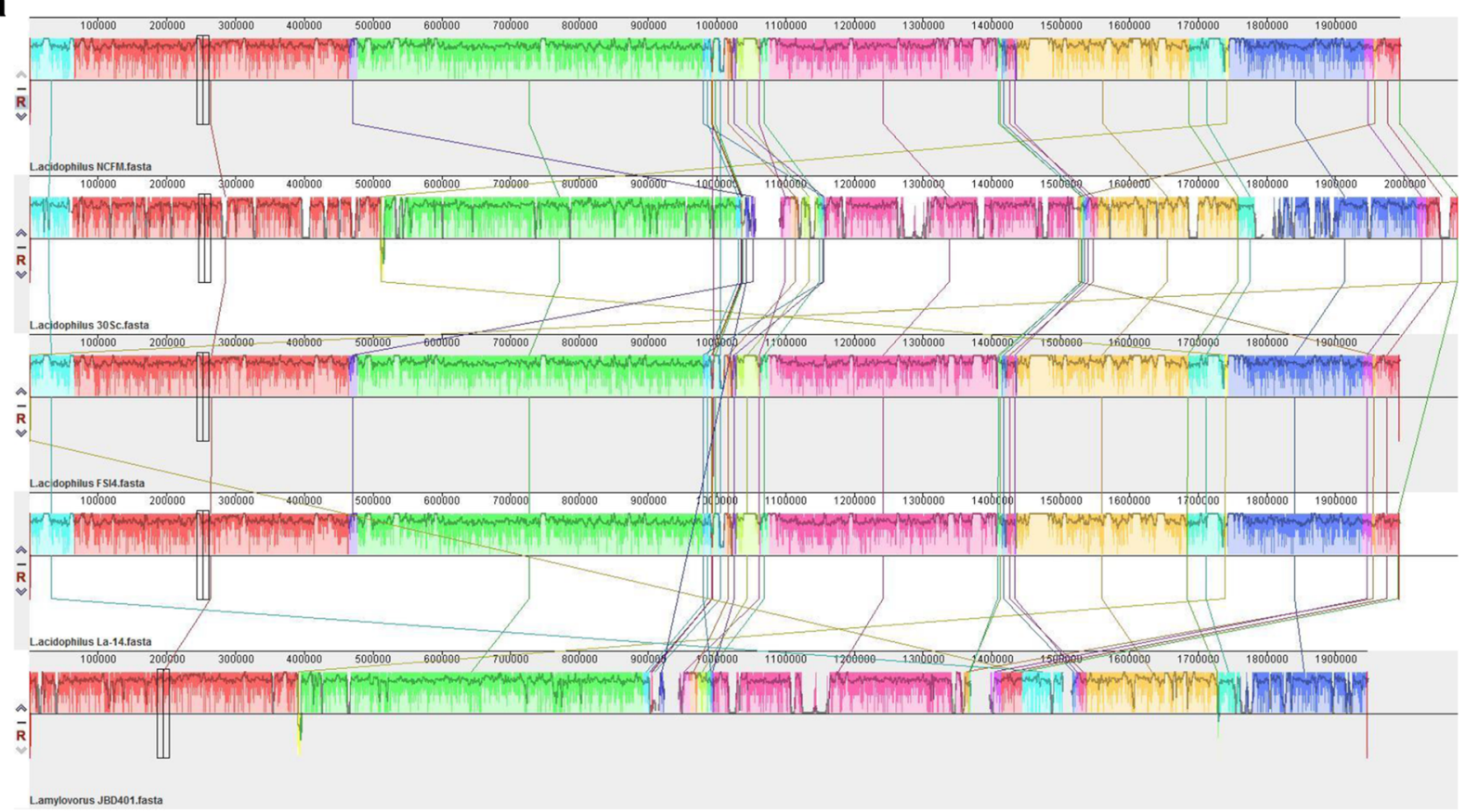

b NCFM and JBD401

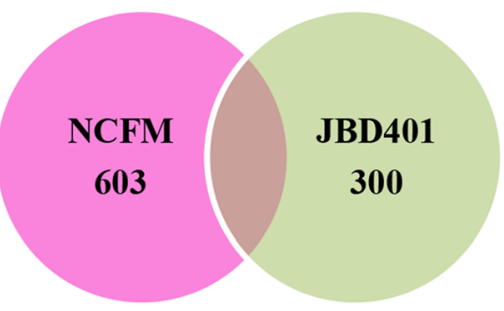

e

FSI4 and JBD401

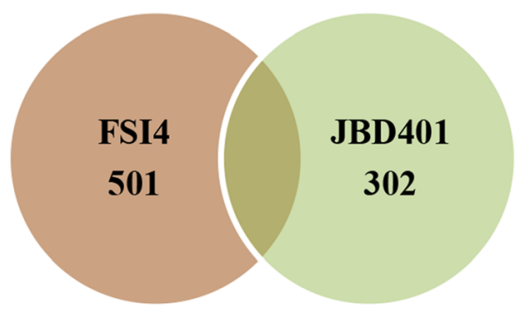

C La-14 and JBD401

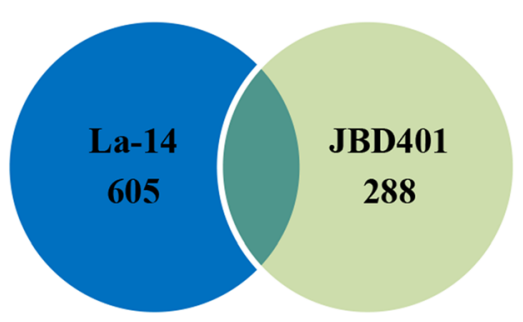

d 30SC and JBD401

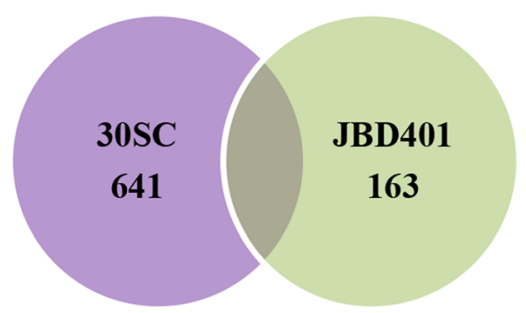

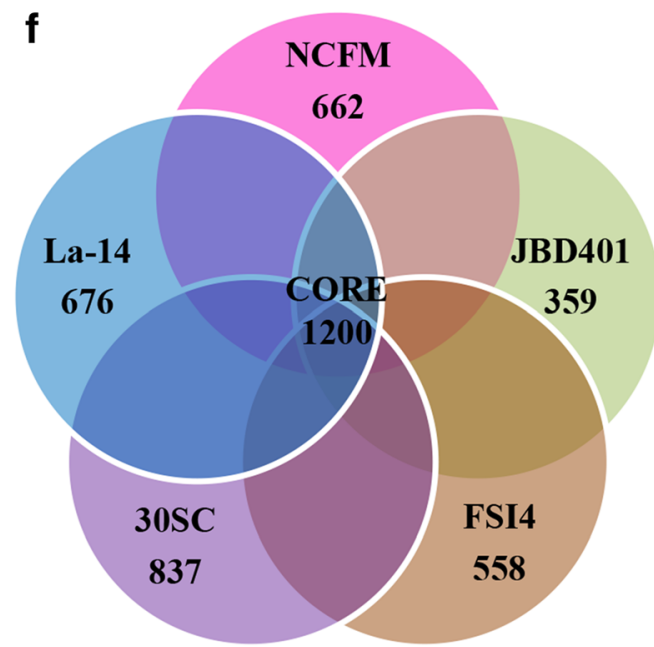

Fig. 4 (See legend on next page.) 
(See figure on previous page.)

Fig. 4 Comparative genome analysis of Lactobacillus amylovorus JBD401 with Lactobacillus acidophilus strains. a Multiple genome alignment of L. acidophilus NCFM, L. acidophilus 30SC, L. acidophilus FSI4, L. acidophilus La-14 and L. amylovorus JBD401 from top to bottom. Image was generated by Mauve. A scale is showing name of each genome sequence compared. The similar colored blocks of each genome are connected through lines representing homologous regions of each genome and non-homologous regions unique to each genome are white. $\mathbf{b}$ Core genes between L. acidophilus NCFM and L. amylovorus JBD401. c Core genes between L. acidophilus La-14 and L. amylovorus JBD401. $\mathbf{d}$ Core genes between L. acidophilus 30SC and L. amylovorus JBD401. e Core genes between L. acidophilus FSI4 and L. amylovorus JBD401. f Core genes among five genomes; L. acidophilus NCFM, L. acidophilus La-14, L. acidophilus 30SC, L. acidophilus FSI4, and L. amylovorus JBD401. 1200 proteins were identified common to all of the strains along with unique genes of each genome; NCFM (662, 64.45\%), La-14 (676, 63.97\%), 30SC (837, 58.91\%), FSI4 (558, 68.26\%) and JBD401 $(359,76.97 \%)$

\section{Discussion}

It is well known that many metabolic disturbances lead to hyperammonemia. Primary hyperammonemia is a genetic disease caused by mutation in the genes required for the urea cycle. There are six types of urea cycle disorder: N-acetylglutamate synthase deficiency; carbamoyl phosphate synthetase-1 deficiency; ornithine carbamoyltransferase deficiency; argininosuccinic acid synthetase deficiency (citrullinemia); argininosuccinase acid lyase deficiency (argininosuccinic aciduria); and arginase deficiency (argininemia) [31]. Primary hyperammonemia has recently attracted attention because of reports that an estimated $20 \%$ of cases of sudden infant death syndrome might to be due to an undiagnosed urea cycle disorder [32]. Secondary hyperammonemia is induced by metabolic defects characterized by reduced activity in enzymes that are not involved in the urea cycle, and various genetic or complex diseases such as propionic acidemia, methylmalonic acidemia, liver cirrhosis, or hepatic failure can induce secondary hyperammonemia [33]. Despite the fact that combined cases of primary and secondary hyperammonemia are frequently noted, a $30 S C$ and JBD401

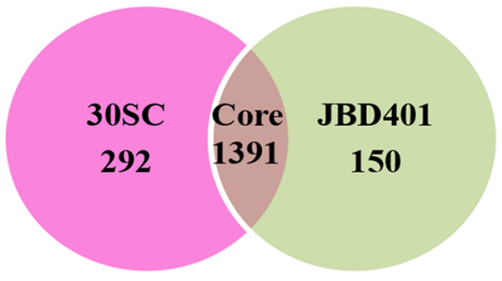

b GRL1118 and JBD401

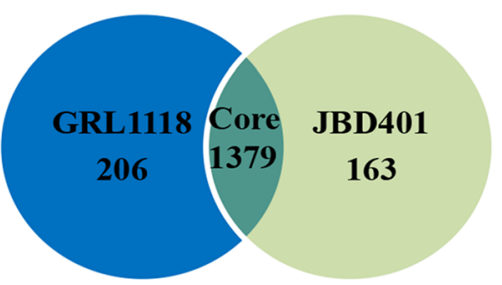

C DSM20531 and JBD401

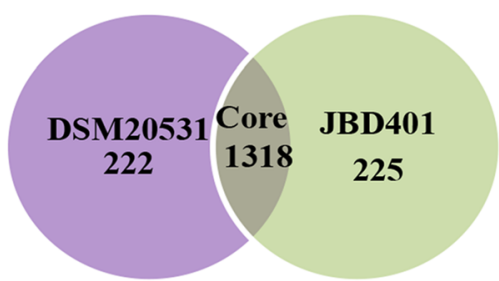

\section{d Blast alignment of Carbamate kinase gene (CK)}

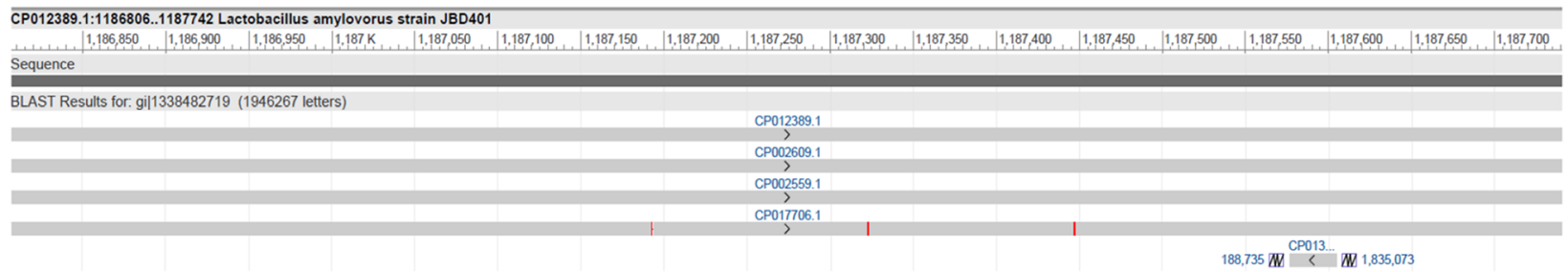

e Blast alignment of ornithine carbamoyltransferase gene (OTC)

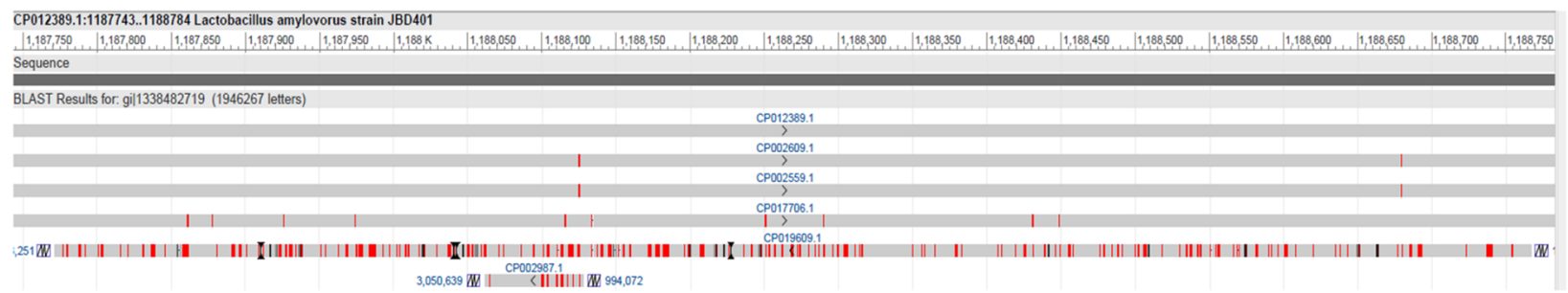

Fig. 5 Comparative genome analysis of Lactobacillus amylovorus JBD401 with Lactobacillus amylovorus strains. a Core genes between L. amylovorus 30SC and L. amylovorus JBD401. b Core genes between L. amylovorus GRL1118 and L. amylovorus JBD401. c Core genes between L. amylovorus DSM20531 and L. amylovorus JBD401. d Blast alignment of carbamate kinase gene (1,186,806 bp-1,187,742 bp) of L. amylovorus JBD401. e Blast alignment of ornithine carbamoyltransferase gene $(1,187,743 \mathrm{bp}-1,188,784 \mathrm{bp})$ of $L$. amylovorus JBD401. d, e red bars in the alignment show mismatches and black bars show gaps 


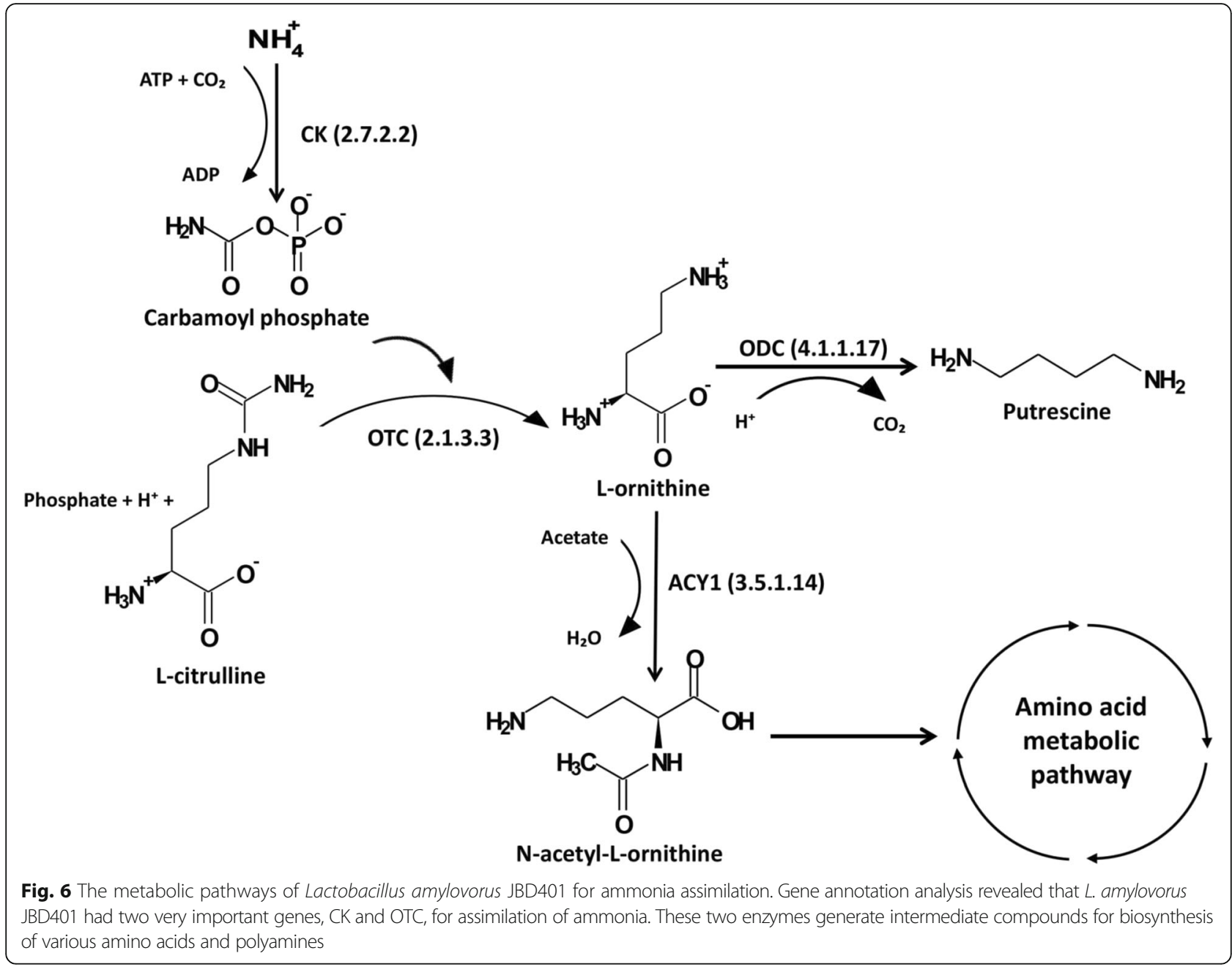

there is no effective medication to treat hyperammonemia [34]. Considering recent advances that metabolic activities of gut microbiota in the intestine are associated with various diseases [35, 36], removal of ammonia by an intestinal bacterium represents a very promising approach for a therapeutic agent for hyperammonemia.

This work identified a Lactobacillus strain that effectively reduced blood ammonia levels in mice through the complete pathways for assimilation of ammonia. $L$. amylovorus JBD401 possessed two very important genes, $\mathrm{CK}$ and OTC. The enzymes encoded by these genes are required for assimilation of ammonia to synthesize Lornithine, which is used as an intermediate compound for biosynthesis of various nitrogen-containing metabolites. L. amylovorus JBD401 also possessed the genes for $\mathrm{N}$-acyl-L-amino acid amidohydrolase (EC 3.5.1.14) at AB283_1076 and N-acetyl-L, L-diaminopimelate aminotransferase (EC 2.6.1.) at AB283_0863 for biosynthesis of L-glutamate from L- ornithine. Gene annotation analyses showed that $L$. amylovorus JBD401 has a complete set of genes for bioconversion of L-glutamate to various amino acids, including glutamine synthetase type I (EC 6 . 3.1.2) at AB283_1841, aspartate aminotransferase (EC 2.6. 1.1) at AB283_0491/0739/0740, N-acyl-L-amino acid amidohydrolase (EC 3.5.1.14) at AB283_1076, and N-acetylL,L-diaminopimelate aminotransferase (EC 2.6.1) at AB283_0863 (Fig. 6). L. amylovorus JBD401 also possesses the gene for ornithine decarboxylase (EC 4.1.1.17) at AB283_1019 for decarboxylation of L-ornithine to biosynthesize putrescine. Putrescine is a key intermediate metabolite for entry into the polyamine metabolic pathway. The presence of complete biosynthetic pathways for various amino acids and polyamine metabolites in the $L$. amylovorus JBD401 genome indicates that $L$. amylovorus JBD401 should actively absorb free amine from its surrounding environment for assimilation into various amino acids and polyamines. Assimilation of free amine into various amino acids and polyamines in the intestinal lumen of the host will reduce ammonia concentrations, resulting in traversing ammonia from the host body to the intestinal lumen to reduce blood ammonia levels. 
Interestingly, the genome of $L$. amylovorus JBD401 contained 372 pseudogenes constituting $15.82 \%$ of the genome. Prokaryotic organisms usually have a very well organized single circular chromosome, which minimizes the content of intergenic nucleotide sequences. Generally $L$. acidophilus strains contain a small number of pseudogenes [22-25]. The higher number of pseudogenes in L. amylovorus JBD401 suggests that this strain might be in an active stage of evolution. During evolution process, L. amylovorus JBD401 seemed to acquire a complete set of genes to assimilate ammonia into various amino acids and polyamines.

The potential usefulness of probiotics in improving the symptoms of neurological diseases such as dementia, Reye syndrome, and Alzheimer's disease is becoming a topic of great interest [37-39]. Especially, many epidemiological studies have repeatedly shown that higher adherence to Mediterranean diet was associated with lower risk of Alzheimer's disease [40, 41]. Although the beneficial effects of probiotics on neurological diseases are becoming apparent recently, the underlying protective mechanism has remained unknown. Considering that ammonia is especially toxic to neurons [42], it would be logical to propose that a reduction of blood ammonia level has a beneficial effect in patients with neurological diseases. In this context, recent animal experiments showing that administration of Lactobacillus strains efficiently reduces blood ammonia levels have shed light on the question of how probiotics exert protective activity against neurological diseases.

Recent experiments clearly showed that administration of some Lactobacillus strains could be used to treat hyperammonemia patients [43-45]; however, their efficacies in reduction of blood ammonia levels were quite variable. In this study, we identified the best probiotic strain for treatment of hyperammonemia. Our work not only explains why Lactobacillus strains showed excellent effects in treatment of hyperammonemia, but also explains the mechanism of the Lactobacillus-assisted neuroprotective effect. In addition, this work will guide future screens to identify potential probiotic strains for treatment of hyperammonemia.

\section{Conclusions}

High throughput screening of probiotic strains followed by subsequent animal experiments resulted in identification of an ideal strain for hyperammonemia, L. amylovorus JBD401. Comparative genomic analysis showed that L. amylovorus JBD401 had a complete set of genes required for ammonia assimilation into various amino acids and polyamines to reduce the blood ammonia levels of the host, providing an explanation for this effect of L. amylovorus JBD401 for hyperammonemia. The genome analysis confirmed that $L$. amylovorus JBD401 contains unusually large number of various pseudogenes suggesting an active stage of evolution which could explain the acquired functionality of ammonia assimilation. This work not only identifies the ideal strain but also explains the underlying mechanism on how a Lactobacillus strain could be used to treat hyperammonemia.

\section{Methods \\ High throughput screening of ammonia-removing probiotic strains}

The probiotic strains were obtained from the Gut Microbiota bank (https://www.gutmicrobiotabank.com) consisting of 23,689 strains. All strains were grown in De Mann Rogosa Sharpe (MRS) broth (Difco ${ }^{\mathrm{TM}}$ ) and maintained on MRS agar plates with routine subculturing at a regular interval of 15 days. For high throughput screening, the probiotic strains were first grown anaerobically in MRS broth at $37{ }^{\circ} \mathrm{C}$ until O.D. range from 0.45 to 1.0 at $600 \mathrm{~nm}$, and $500 \mu \mathrm{L}$ of each culture was transferred into 96 well plates. After addition of $10 \mu \mathrm{L}$ of ammonium hydroxide $(30 \mu \mathrm{g} / \mathrm{mL})$ to the 96 well plates, the cultures were incubated anaerobically at $37{ }^{\circ} \mathrm{C}$ for $30 \mathrm{~min}$ and then centrifuged at $4000 \mathrm{rpm}$ for $15 \mathrm{~min}$ at $4{ }^{\circ} \mathrm{C}$. After centrifugation, the supernatants in the 96 well plates were transferred into new 96 well plates, and the ammonia concentrations in each supernatant were measured by an ammonia assay kit (AA0100; Sigma-Aldrich) according to manufacturer's protocol.

\section{Measuring blood and brain ammonia levels and caspase induction in test animals}

In vitro high throughput screening resulted in identification of L. amylovorus JBD401 removing free ammonia in its surrounding MRS medium. To evaluate the efficacy of L. amylovorus JBD401 to hyperammonemia, $1 \times 10^{7} \mathrm{CFU}$ of $L$. amylovorus JBD401 culture was orally administered to four-week-old male ICR mice for 7 days. Hyperammonemia was induced by intravenous administration of $100 \mathrm{mg} / \mathrm{kg}$ of $\mathrm{NH}_{4} \mathrm{Cl}$ into the tail on the last day. Fifty minutes after injection of ammonia, blood was collected, and the brain was removed. Brain tissue collection procedure was initiated after animals had been euthanized by cervical dislocation. All efforts were made to minimize suffering. The blood was centrifuged to collect supernatant serum for measurement of ammonia. The brains were ground using a homogenizer, and the supernatants of the ground brains were used to quantitate ammonia concentrations. The ammonia concentrations in each supernatant samples were measured by an ammonia assay kit (AA0100; Sigma-Aldrich) according to manufacturer's protocol at 200-300 nm using a nanodrop machine (Thermo Scientific). 
Caspase induction was measured using a FluorescentLabeled Inhibitor of Caspases (FLICA ${ }^{\mathrm{sm}}$ ) assay kit. Brain tissue of mouse was sliced using a cryotome (Thermo Scientific). The FLICA Caspase 9 reagent was reconstituted with $50 \mu \mathrm{L}$ of DMSO to form the stock concentrate, which was diluted with $200 \mu \mathrm{L}$ PBS to give the working solution. The working solution was directly added to samples and controls at a ratio of 1:30 to 1:60 and incubated for 15 to $45 \mathrm{~min}$. Cells were washed and centrifuged two or three times. Cells were labeled with Hoechst stain or DAPI and/or fixed with fixative included in the assay kit. Data were analyzed using a fluorescence microscope (Nikon).

\section{Genome sequencing and assembly}

The L. amylovorus JBD401 (KCTC 11515BP) genome was sequenced by next-generation sequencing using 454 (454 GS FLX) and Illumina methods. Initially, 454 DNA reads were assembled into contigs with the PHRED/ PHRAP/CONSED [46] software package and subcontracted to Macrogen Inc. Further contigs were assembled using Geneious pro.5.5 software [47] and CLC workbench software (CLC Bio-Qiagen). Mapping of assembled contigs was performed by Projector 2 software [48]. Gaps were closed by adopting a PCR-based strategy. Ends of scaffolds were used for primer design, and the inter-scaffold region was amplified by sequencing multiplex PCR products using a long-range PCR kit (Qiagen). Primer3 software [49] was used to design primers, and primer synthesis and PCR product sequencing were performed at Xenotech.

\section{Genome annotation}

The complete genome sequence was subjected to an automated annotation process by RAST [50]. Quality of reads was examined by FastQC [51] (Additional file 1: Figure S2), and contigs were ordered against a reference, Lactobacillus amylovorus GRL1118, using Mauve (multiple genome alignment) (Additional file 1: Figure S1) [52]. According to the RAST server, the genome sequence of $L$. amylovorus JBD401 contains 230 subsystems. Every subsystem is a collection of functional roles and associated to each other in a system, for example, a metabolic pathway system. The genome was further curated manually using web-based software and databases. FrameD [53] was used for prediction of ORFs, and their starts were manually adjusted based on blast alignment. The gene model was predicted by Glimmer [54] and compared with the following public databases: COGs [55], Interpro [56], Pfam [57], Prints [58], PROSITE [59], Smart [60], Swissprot [61], and Tigrfams [62]. Tblastx [63] was used to reanalyze intergenic regions for ORFs. Hmmer [64] on pfam 5.4 was used for motif analysis. Stringent parameters were used to identify tRNAs by trnascan-se [65] and ribosomal binding sites by RBSfinder. After resequencing, the Genome atlas was generated by Geneious 5.5 and PATRIC [66]. Prophage regions within the genome were identified using PHAST. Whole-genome nucleotide alignments were generated and visualized by Mauve. Identification of core genes and unique genes was carried out by coregenes 3.0.

\section{In silico analysis of antibiotic resistant genes in genome of L. amylovorus JBD401}

The presence of homologs of various antibiotic resistance genes in the genome of $L$. amylovorus JBD401 was checked by performing BLASTp searches of the entire genome (under multiple gene annotation category) against the ARDB database [67]. We used identity threshold of $40 \%$ which is also a default search criteria in ARDB database. Similarly, a previous study have also used relaxed identity threshold of $35 \%$ to identify antibiotic resistance genes in swine gut metagenomes [68].

\section{Functional analysis}

Gene ontology (GO) analysis was carried out using Blast2GO basic account to analyze the FASTA-formatted sequences of predicted genes of $L$. amylovorus JBD401 genome into the three GO categories of biological process, molecular function, and cellular component. Protein sequences of $L$. amylovorus JBD401 were annotated with blastp followed by Blast2GO mapping and GO annotation. This provided vocabularies from GO terms, enzyme codes (EC), InterPro IDs, and KEGG pathways. Homologous sequences were identified by blast and mapped to GO terms that were associated with blast hits. Mapping of GO terms was reduced by GO-slim ontology. Multiple testing in Blast2GO was performed by Fisher's exact test to compute GO significance levels.

\section{Additional file}

Additional file 1: Annotation and organization of L. amylovorus JBD401 genome in this study. (DOCX $4200 \mathrm{~kb}$ )

\section{Abbreviations}

A: Aspartic; ANI: Average nucleotide identity; Asp: Aspartic acid; BP: Biological process; C: Cysteine; CC: Cellular component; CK: Carbamate kinase;

Cys: Cysteine; EC: Enzyme codes; FLICA ${ }^{\text {TM}}$. Fluorescent-labeled inhibitor of caspases; FOS: Fructooligosaccharides; Gln: Glutamine; Glu: Glutamic acid; Gly: Glycine; GO: Gene ontology; HGT: horizontal gene transfer; L.: Lactobacillus; M: Metallo; MF: Molecular function; MRS: De Mann Rogosa Sharpe; OTC: Ornithine carbamoyltransferase; RhBG: (Rh family, B glycoprotein); RhCG: (Rh family, C glycoprotein); S: Serine; Ser: Serine; T: Threonine; Thr: Threonine; VTEC: verocytotoxin-producing Escherichia coli

\section{Acknowledgements}

This work was supported by the Technology Development Program (S2562170) funded by the Ministry of SMEs and Startups (MSS, Korea).

\section{Funding}

This research was supported by the Technology Development Program for Agriculture and Forestry No. 313040-3, Ministry for Food, Agriculture, 
Forestry and Fisheries, Republic of Korea. This funding body had no role in the design of the study and collection, analysis, and interpretation of data, or in writing the manuscript.

\section{Availability of data and materials}

The complete sequence of L. amylovorus JBD401 and annotation reported in this paper has been deposited in GenBank under Accession No. CP012389.

\section{Authors' contributions}

Conceived and designed the experiments and wrote the paper: S.T.H. and H.J.K. Performed the experiments: P.S., I.A.L. and R.D. Analyzed the data: P.S. S.T.H., H.J.K., and H.J.C. All authors read and approved the final manuscript.

\section{Ethics approval}

Animals in this study, four-week-old male ICR mice were purchased from Joongang Experimental Animal Co., Seoul, Korea. All animal care and use protocols were performed strictly in accordance with the ethical guidelines of the Ethics Committee of the Chonbuk National University Laboratory Animal Center, and the animal study protocol was approved by the Ethics Committee of the Chonbuk National University Laboratory Animal Center (Approved Number: CBU 2012-0040) in accordance with the "Guide for the Care and Use of Laboratory Animals," published by the National Research Council and endorsed by the NIH Office of Laboratory Animal Welfare.

\section{Competing interests}

The authors declare that they have no competing financial interests.

\section{Publisher's Note}

Springer Nature remains neutral with regard to jurisdictional claims in published maps and institutional affiliations.

\section{Author details}

'Department of Biomedical Sciences and Institute for Medical Science, Chonbuk National University Medical School, Jeonju, Chonbuk 54907, South Korea. ${ }^{2}$ JINIS BDRD institute, JINIS Biopharmaceuticals Co., 913 Gwahak-Ro, Bongdong, Wanju, Chonbuk 55321, South Korea. ${ }^{3}$ Present address: Department of Chemistry, Gunsan National University, Gunsan, Chonbuk 51450, South Korea.

\section{Received: 23 November 2017 Accepted: 13 April 2018}

\section{Published online: 25 April 2018}

\section{References}

1. Lockwood AH, McDonald JM, Reiman RE, Gelbard AS, Laughlin JS, Duffy TE, et al. The dynamics of ammonia metabolism in man. Effects of liver disease and hyperammonemia. J Clin Invest. 1979;63:449-60.

2. Blei AT, Cordoba J. Hepatic encephalopathy. Am J Gastroenterol. 2001;96: 1968-76.

3. Bachmann C. Mechanisms of hyperammonemia. Clin Chem Lab Med. 2002; 40:653-62.

4. Al Sibae MR, McGuire BM. Current trends in the treatment of hepatic encephalopathy. Ther Clin Risk Manag. 2009;5:617-26.

5. Fisman M, Gordon B, Feleki V, Helmes E, Appell J, Rabheru K. Hyperammonemia in Alzheimer's disease. Am J Psychiatry. 1985;142:71-3.

6. Branconnier RJ, Dessain EC, McNiff ME, Cole JO. Blood ammonia and Alzheimer's disease. Am J Psychiatry. 1986;143:1313-4.

7. Mendiola-Precoma J, Berumen LC, Padilla K, Garcia-Alcocer G. Therapies for prevention and treatment of Alzheimer's disease. Biomed Res Int. 2016:2016:1-17.

8. Bonfili L, Cecarini V, Berardi S, Scarpona S, Suchodolski JS, Nasuti C, et al. Microbiota modulation counteracts Alzheimer's disease progression influencing neuronal proteolysis and gut hormones plasma levels. Sci Rep. 2426;2017:7

9. Xu J, Gordon J. Honor thy symbionts. Proc Natl Acad Sci U S A. 2003;100: 10452-9.

10. Gorbach SL. Medical microbiology: microbiology of the gastrointestinal tract. In: Baron S. Galveston, editor. The University of Texas Medical Branch at Galveston; 1996

11. Worrell RT, Merk L, Matthews JB. Ammonium transport in the colonic crypt cell line,T84:role for rhesus glycoproteins and NKCC1. Am J Physiol Gastrointest Liver Physiol. 2008;294:G429-40.
12. Wrong $\mathrm{OM}$, Vince $\mathrm{A}$. Urea and ammonia metabolism in the human large intestine. Proc Nutr Soc. 1984:43:77-86.

13. Sanz Y, De Palma G. Gut microbiota and probiotics in modulation of epithelium and gut associated lymphoid tissue function. Int Rev Immunol. 2009;28:397-413.

14. Nicaise C, Prozzi D, Viaene E, Moreno C, Gustot T, Quertinmont E, et al. Control of acute, chronic, and constitutive hyperammonemia by wild-type and genetically engineered lactobacillus plantarum in rodents. Hepatology. 2008:48:1184-92.

15. Shen TD, Albenberg L, Bittinger $K$, Chehoud $C$, Chen $Y Y$, Judge $C A$, et al. Engineering the gut microbiota to treat hyperammonemia. J Clin Invest. 2015;125:2841-50.

16. Suzuki H, Yanaka A, Shibahara T, Matsui H, Nakahara A, Tanaka N, et al. Ammonia-induced apoptosis is accelerated at higher $\mathrm{pH}$ in gastric surface mucous cells. Am J Physiol Gastrointest Liver Physiol. 2002;283:G986-95.

17. Zago M, Scaltriti E, Rossetti L, Guffanti A, Armiento A, Fornasari ME, et al. Characterization of the genome of the dairy lactobacillus helveticus bacteriophage AAQ113. Appl Environ Microbiol. 2013;79:4712-8.

18. Mahony J, van Sinderen D. Current taxonomy of phages infecting lactic acid bacteria. Front Microbiol. 2014;5:1-7.

19. Joensen KG, Scheutz F, Lund O, Hasman H, Kaas RS, Nielsen EM, et al. Real time whole-genome sequencing for routine typing, surveillance, and outbreak detection of verotoxigenic Escherichia coli. J Clin Microbiol. 2014; $52: 1501-10$

20. Schonert S, Buder T, Dahl MK. Properties of maltose-inducible alphaglucosidase MalL (sucrase-isomaltase-maltase) in Bacillus subtilis: evidence for its contribution to maltodextrin utilization. Res Microbiol. 1999;150:167-77.

21. Han N, Qiang YJ, Zhang W. ANItools web: a web tool for fast genome comparison within multiple bacterial strains. Database-Oxford. 2016;2016: baw084.

22. Altermann E, Russell WM, Azcarate-Peril MA, Barrangou R, Buck BL, McAuliffe $\mathrm{O}$, et al. Complete genome sequence of the probiotic lactic acid bacterium lactobacillus acidophilus NCFM. Proc Natl Acad Sci U S A. 2005;102:3906-12.

23. Oh S, Roh H, Ko HJ, Kim S, Kim KH, Lee SE, et al. Complete genome sequencing of lactobacillus acidophilus 30SC, isolated from swine intestine. J Bacteriol. 2011;193:2882-3.

24. Stahla B, Barrangou R. Complete genome sequence of probiotic strain lactobacillus acidophilus La-14. Genome Announc. 2013;1:e00376-13.

25. lartchouk O, Kozyavkin S, Karamychev V, Slesarev A. Complete genome sequence of lactobacillus acidophilus FSI4, isolated from yogurt. Genome Announc. 2015;3:e00166-15.

26. Kant R, Paulin L, Alatalo E, de Vos WM, Palva A. Genome sequence of lactobacillus amylovorus GRL1118, isolated from pig ileum. J Bacteriol. 2011; 193:3147-8.

27. Lerat $\mathrm{E}$, Ochman H. Psi-phi: exploring the outer limits of bacterial pseudogenes. Genome Res. 2004;14:2273-8.

28. Liu Y, Harrison PM, Kunin V, Gerstein M. Comprehensive analysis of pseudogenes in prokaryotes: widespread gene decay and failure of putative horizontally transferred genes. Genome Biol. 2004:5:R64.

29. Zafar N, Mazumder R, Seto D. CoreGenes: a computational tool for identifying and cataloging "core" genes in a set of small genomes. BMC Bioinf. 2002:3:12

30. Gotz S, Garcia-Gomez JM, Terol J, Williams TD, Nagaraj SH, Nueda MJ, et al. High-throughput functional annotation and data mining with the Blast2GO suite. Nucleic Acids Res. 2008:36:3420-35.

31. Braissant $\mathrm{O}$. Current concepts in the pathogenesis of urea cycle disorders. Mol Genet Metab. 2010;100(Suppl 1):S3-S12

32. Burton BK. Inborn errors of metabolism in infancy: a guide to diagnosis. Pediatrics. 1998;102:E69.

33. Haberle J. Clinical and biochemical aspects of primary and secondary hyperammonemic disorders. Arch Biochem Biophys. 2013;536:101-8.

34. Häberle J, Boddaert N, Burlina A, Chakrapani A, Dixon M, Huemer M, et al. Suggested guidelines for the diagnosis and management of urea cycle disorders. Orphanet J Rare Dis. 2012;7:32.

35. Bajaj JS, Hylemon PB, Younossi Z. The intestinal microbiota and liver disease. Am J Gastroenterol Suppl. 2012:1:9-14.

36. Chung HJ, Yu JG, Lee IH, Liu MJ, Shen YF, Sharma SP, et al. Intestinal removal of free fatty acids from hosts by lactobacilli for the treatment of obesity. FEBS Open Bio. 2016;6:64-76.

37. Alkasir R, Li J, Li X Jin M, Zhu B. Human gut microbiota: the links with dementia development. Protein Cell. 2017;8:90-102. 
38. Umbrello G, Esposito S. Microbiota and neurologic diseases:potential effects of probiotics. J Transl Med. 2016;14:1-11.

39. Wang J, Tang H, Zhang C, Zhao Y, Derrien M, Rocher E, et al. Modulation of gut microbiota during probiotic-mediated attenuation of metabolic syndrome in high fat diet-fed mice. ISME J. 2015;9:1-15.

40. Del Chierico F, Vernocchi P, Dallapiccola B, Putignani L. Mediterranean diet and health: food effects on gut microbiota and disease control. Int J Mol Sci. 2014;15:11678-99.

41. Scarmeas N, Stern Y, Tang MX, Mayeux R, Luchsinger JA. Mediterranean diet and risk for Alzheimer's disease. Ann Neurol. 2006;59:912-21.

42. Butterworth RF. Pathophysiology of hepatic encephalopathy: a new look at ammonia. Metab Brain Dis. 2002;17:221-7.

43. McGee RG, Bakens A, Wiley K, Riordan SM, Webster AC. Probiotics for patients with hepatic encephalopathy. Cochrane Database Syst Rev. 2011; 11:805-7.

44. Luo J, Wang T, Liang S, Hu X, Li W, Jin F. Ingestion of lactobacillus strain reduces anxiety and improves cognitive function in the hyperammonemia rat. Sci China Life Sci. 2014;57:327-35.

45. Wallace CJK, Milev R. The effects of probiotics on depressive symptoms in humans: a systematic review. Ann General Psychiatry. 2017;16:1-10.

46. Gordon D, Abajian C, Green P. Consed: a graphical tool for sequence finishing. Genome Res. 1998;8:195-202.

47. Kearse M, Moir R, Wilson A, Stones-Havas S, Cheung M, Sturrock S, et al. Geneious basic: an integrated and extendable desktop software platform for the organization and analysis of sequence data. Bioinformatics. 2012;28: 1647-9.

48. van Hijum SAFT, Zomer AL, Kuipers OP, Kok J. Projector 2: contig mapping for efficient gap-closure of prokaryotic genome sequence assemblies. Nucleic Acids Res. 2005;33:W560-6.

49. Rozen S, Skaletsky H. Primer3 on the WWW for general users and for biologist programmers. Methods Mol Biol. 2000;132:365-86.

50. Aziz RK, Bartels D, Best AA, DeJongh M, Disz T, Edwards RA, et al. The RAST server: rapid annotations using subsystems technology. BMC Genomics. 2008;9:75

51. Andrews S. FastQC a quality control tool for high throughput sequence data. 2010; https://www.bioinformatics.babraham.ac.uk/projects/fastqc/.

52. Darling AC, Mau B, Blattner FR, Perna NT. Mauve: multiple alignment of conserved genomic sequence with rearrangements. Genome Res. 2004;14: 1394-403.

53. Schiex T, Gouzy J, Moisan A, de Oliveira Y. FrameD: a flexible program for quality check and gene prediction in prokaryotic genomes and noisy matured eukaryotic sequences. Nucleic Acids Res. 2003;31:3738-41.

54. Salzberg SL, Delcher AL, Kasif S, White O. Microbial gene identification using interpolated Markov models. Nucleic Acids Res. 1998;26:544-8.

55. Tatusov RL, Fedorova ND, Jackson JD, Jacobs AR, Kiryutin B, Koonin EV, et al. The COG database: an updated version includes eukaryotes. BMC Bioinf. 2003;4:41.

56. Zdobnov EM, Apweiler R. InterProScan-an integration platform for the signature recognition methods in InterPro. Bioinformatics. 2001;17:847-8.

57. Finn RD, Bateman A, Clements J, Coggill P, Eberhardt RY, Eddy SR, et al. Pfam: the protein families database. Nucleic Acids Res. 2014;42:D222-30.

58. Attwood TK, Croning MDR, Flower DR, Lewis AP, Mabey JE, Scordis P, et al. PRINTS-S: the database formerly known as PRINTS. Nucleic Acids Res. 2000; 28:225-7.

59. Sigrist CJA, Cerutti L, de Castro E, Langendijk-Genevaux PS, Bulliard V, Bairoch A, et al. PROSITE, a protein domain database for functional characterization and annotation. Nucleic Acids Res. 2010;38:D161-6.

60. Letunic I, Copley RR, Pils B, Pinkert S, Schultz J, Bork P. SMART 5: domains in the context of genomes and networks. Nucleic Acids Res. 2006;34:D257-60.

61. Bairoch A, Apweiler R. The SWISS-PROT protein sequence data bank and its new supplement TREMBL. Nucleic Acids Res. 1996;24:21-5.

62. Haft DH, Selengut JD, White O. The TIGRFAMs database of protein families. Nucleic Acids Res. 2003;31:371-3.

63. Pertsemlidis A, Fondon JW, $3^{\text {rd }}$. Having a BLAST with bioinformatics (and avoiding BLASTphemy). Genome Biol. 2001;2:2002.1-2002.10.

64. Finn RD, Clements J, Eddy SR. HMMER web server: interactive sequence similarity searching. Nucleic Acids Res. 2011;39:W29-37.

65. Lowe TM, Eddy SR. tRNAscan-SE: a program for improved detection of transfer RNA genes in genomic sequence. Nucleic Acids Res. 1997;25:955-64.
66. Snyder EE, Kampanya N, Lu J, Nordberg EK, Karur HR, Shukla M, et al. PATRIC: the VBI PathoSystems resource integration center. Nucleic Acids Res. 2007;35:D401-6.

67. Liu B, Pop M. ARDB-antibiotic resistance genes database. Nucleic Acids Res. 2009;37:D443-7.

68. Looft T, Johnson TA, Allen HK, Bayles DO, Alt DP, Stedtfeld RD, et al. In-feed antibiotic effects on the swine intestinal microbiome. Proc Natl Acad Sci U S A. 2012;109:1691-6.

\section{Ready to submit your research? Choose BMC and benefit from:}

- fast, convenient online submission

- thorough peer review by experienced researchers in your field

- rapid publication on acceptance

- support for research data, including large and complex data types

- gold Open Access which fosters wider collaboration and increased citations

- maximum visibility for your research: over $100 \mathrm{M}$ website views per year

At BMC, research is always in progress.

Learn more biomedcentral.com/submissions 